\title{
CONCORDANCIA VARIABLE EN ESPAÑOL DE HERENCIA: ESTUDIO DEL GRUPO NOMINAL
}

\section{AGREEMENT VARIABILITY IN SPANISH AS A HERITAGE LANGUAGE: STUDY OF NOMINAL PHRASES}

\author{
Juan Pablo Orejudo \\ Universidad de Salamanca \\ juanpa_og@usal.es \\ https://orcid.org/0000-0001-7436-4104
}

Recibido: 29/11/2019

Aceptado: 28/04/2020

\section{Resumen}

Los hablantes de herencia en la zona norte de Texas tienen español como lengua familiar e inglés como lengua vehicular. El español queda relegado al habla coloquial, dejando al margen el registro formal y la escritura. Por este motivo, la competencia lingüística en español de estos hablantes no es completa y existen problemas concretos con la concordancia dentro de grupos nominales con tres constituyentes (artículo, nombre y adjetivo). En esta investigación se realizaron entrevistas semidirigidas a una muestra de hablantes de herencia de tres generaciones sociolingüísticas (G1, G2 y G3) para crear un corpus oral. Se analizaron un total de 24 entrevistas para contabilizar el número de grupos nominales con tres constituyentes utilizados y relacionarlos con las variables de generación sociolingüística, género del hablante, posición del ítem con respecto al verbo (preverbal, posverbal o aislado) y concordancia interna (género y número). Los resultados obtenidos muestran que el uso de grupos

\begin{abstract}
Heritage speakers in North Texas use the Spanish language as a family language, and English is the vehicular language. Spanish is used for colloquial speech, leaving aside formal register and writing. For this reason, competence in Spanish is not complete and there are specific problems with agreement within nominal phrases with three constituents (article, noun, and adjective). In this research, semi-structured interviews were given to heritage speakers from three sociolinguistic generations (G1, G2, and G3) in order to create an oral corpus. Twenty four interviews were analyzed to count the number of nominal phrases with three constituent in them and their relation to the variables of sociolinguistic generation, speaker's gender, item position related to the verb (pre-verbal, post-verbal, or isolated) and internal agreement (gender and number). Results show that the use of complex nominal phrases decreases while the internal
\end{abstract}

Para citar este artículo / To cite this article: Orejudo, Juan Pablo (2020). Concordancia variable en español de herencia: estudio del grupo nominal. ELUA, 34: 153-173. https://doi.org/10.14198/ ELUA2020.34.7

Enlace / Link: https://doi.org/10.14198/ELUA2020.34.7 
nominales compuestos disminuye mientras que los casos sin concordancia aumentan porcentualmente según crece la generación sociolingüística.

PALABRAS CLAVE: Hablantes de herencia, concordancia variable, grupo nominal, español. disagreement increases in percentage when the sociolinguistic generation goes up.

KEYWORDS: Heritage speaker, agreement variability, nominal phrase, Spanish.

\section{INTRODUCCIÓN}

En este artículo se investiga la concordancia de grupos nominales de tres constituyentes (determinante, nombre y adjetivo) en español como lengua de herencia con énfasis en la zona norte de Texas. Se analiza la concordancia en la lengua de herencia para mostrar en qué momento del aprendizaje podría introducirse su enseñanza, es decir, responder a la pregunta del cuándo. El objetivo de la investigación es encontrar la norma que subyace al concordar en español como lengua de herencia. Si se reconocen patrones de uso y se sabe cuándo y cómo se concuerdan los grupos nominales, se podrá mejorar su enseñanza y suplir las necesidades educativas que existen actualmente. En esta investigación se espera la confirmación de cuatro hipótesis:

1. Uso decreciente de grupos nominales de tres constituyentes (determinante, sustantivo y adjetivo) según aumenta la generación sociolingüística.

2. Descenso porcentual de concordancia según aumenta la generación sociolingüística.

3. Predominancia de uso posverbal de estos grupos nominales.

4. Igualdad de resultados entre hombres y mujeres.

\section{CONCEPTOS PREVIOS}

Los conceptos que requieren clarificación previa son: lengua de herencia, grupo nominal y concordancia.

\subsection{Hablante de herencia}

Para comprender el concepto de lengua de herencia debe definirse qué es un hablante de herencia. Valdés (2001: 38) define hablante de herencia (HH) como aquella persona que ha crecido en un ambiente donde no se habla inglés, aunque lo habla o lo entiende, teniendo competencia bilingüe (habilidades lingüísticas) en la lengua familiar y en inglés. En otras palabras, un $\mathrm{HH}$ es el individuo que tiene dos lenguas, independientemente de su competencia lingüística en ellas: una que habla en casa y es minoritaria; y otra que habla fuera de casa y es la lengua vehicular del país. Según Potowski y Escobar (2015: IX), Estados Unidos es el segundo país del mundo con más hablantes de español de herencia y la cifra aumenta día a día. En este contexto, el español sería la lengua familiar o de herencia y el inglés sería la lengua mayoritaria o vehicular. El español de los hablantes de herencia en Estados Unidos, transferido por vía familiar gracias en gran medida al importante rol que juegan las madres en su desarrollo (Potowski 2018: 12), posee características lingüísticas concretas dependiendo de la zona geográfica. No obstante, independientemente del lugar en 
el que se encuentre, las características comunes más importantes del habla de herencia son: alternancia de código (Lipski 2005; Domitrescu 2014; Fairchild y Van Hell 2015); uso de calcos y préstamos lingüísticos tanto en el plano léxico como en el morfosintáctico (Moreno 2009; Miller y Gutiérrez, 2019); y patrones fonéticos y fonológicos propios tanto en la prosodia como en sonidos vocálicos y consonánticos (Ronquest y Rao 2018).

Esta investigación se centra en la zona norte de Texas, cuarto estado de los Estados Unidos con mayor población mexicana según la Encuesta de la Comunidad Estadounidense de la Oficina del Censo de los Estados Unidos ${ }^{1}$. Cervantes y Rodríguez (2015: 24) estudiaron los datos demográficos de la zona para concluir que la escolaridad de la población mexicana inmigrante en Estados Unidos es baja. En 2013, del grupo de inmigrantes mexicanos mayores de 25 años, solo el 5.8\% contó con licenciatura completa o más, mientras que tal porcentaje para el total de inmigrantes fue del $28.2 \%$.

Los hablantes de herencia se clasifican por generaciones sociolingüísticas dependiendo del origen, edad de llegada a los Estados Unidos y tiempo de contacto con la comunidad mayoritaria. Montrul (2015: 42-43) defiende que, aunque los hablantes muestren patrones de competencia lingüística similares, no existe un mismo baremo para todos los HH porque cada uno posee una capacidad bilingüe diferente. De esta forma, se pueden encontrar dos individuos de la misma generación sociolingüística con patrones lingüísticos similares pero diferente competencia en español. La mayor parte de la adquisición de la LH proviene de pautas naturales y procesos no formales que se van perdiendo desde la primera generación hasta su desaparición, generalmente a partir de la tercera generación. Esta clasificación se relaciona directamente con la competencia lingüística de los hablantes. Según Potowski y Escobar (2015: 22):

1. La primera generación (G1) llegó a EE.UU. con más de 9 años de edad y el español es su lengua nativa. El inglés es la nueva lengua y la competencia dependerá del tiempo que permanezca en el país.

2. La segunda generación (G2) nació en EE.UU. y tiene dos padres G1. El español es una lengua fuerte pero el inglés es la dominante.

3. La tercera generación (G3) nació en EE.UU. y sus padres son G2. El inglés es la lengua dominante y tienen menos competencia en español.

De esta forma, se observa un patrón general en el que los hablantes de la G1 tiene una competencia en español más alta que los hablantes de la G2, y esta última la tiene más alta que los hablantes de la G3 (Potowski y Escobar 2015: 25).

\subsection{Grupo nominal}

Entendemos como grupo nominal a la estructura gramatical formada por un sustantivo o nombre y otras palabras que lo acompañan, como adjetivos y determinantes:

1. Según el DLE (2014), los determinantes son palabras que generalmente se colocan delante del nombre y lo definen. Laca (1999: 893) completa esta definición explicando que los determinantes son una clase semántica que «forman con el nombre al que

1 https://www.census.gov/quickfacts/fact/table/TX/POP010210 
preceden una expresión referencial o una expresión cuantificadora». En los siguientes ejemplos el determinante está en cursiva, el gato blanco, aquellos muebles amarillos, la silla alta, estas gafas sucias. Los casos de determinante en posición posnominal no son comunes y suelen utilizarse para enfatizar el sustantivo: vamos al sitio aquel.

2. El DLE (2014) define nombre como aquellas palabras que identifican seres animados o inanimados. Bosque (1999: 5) amplia diciendo que el nombre «es la categoría gramatical que expresa la pertenencia de las cosas a alguna clase». Así, al utilizar el nombre libro para referirnos a un objeto que está sobre la mesa, lo identificamos como perteneciente a la categoría o familia de los libros. Algunos ejemplos de nombres, marcados en cursiva, son: el gato blanco, los muebles amarillos, la silla alta, las gafas sucias.

3. En el DLE (2004), los adjetivos son definidos como palabras que indican una cualidad o accidente. Por su parte, Demonte (1999: 134) añade que son palabras graduables y medibles que acompañan a otras para dotarlas de propiedades y delimitar, caracterizar o identificar su naturaleza. Por ejemplo, el gato blanco, los muebles amarillos, la silla alta, las gafas sucias.

El uso de grupos nominales con varios constituyentes es abundante para referirnos a referentes concretos puesto que, como explica Rigau (1999: 311), los modificadores que aparecen con los nombres les dan propiedades específicas para referenciarlos, desarrollar sus propiedades o cuantificarlos. Si estos modificadores no estuvieran, el significado de la construcción sería diferente y el nombre llamaría a otra realidad.

\subsection{Concordancia}

En el segundo tomo del manual de Gramática descriptiva de la lengua española, Martínez (1999: 2697) define concordancia como la «relación entre al menos dos palabras que se establece con la repetición en cada una de ellas de uno de los morfemas de género, de número $[\ldots]$ o de persona, y que sirve en lo fundamental para relacionar e identificar léxica y sintácticamente las palabras concordantes, entre otras, artículo [...] y adjetivo [...] con sustantivo». El DLE (2014) simplifica la definición previa al indicar que concordancia es «la coincidencia obligada de determinados accidentes gramaticales (género, número y persona) entre distintos elementos variables de la oración». Con estas dos definiciones se evidencia que la relación existente entre el determinante, el adjetivo y el sustantivo es obligatoria y sirve para marcarlos tanto léxica como sintácticamente. Se pueden distinguir dos tipos de concordancia: nominal y verbal, aunque este estudio se centra solo en la nominal.

1. Concordancia nominal (coincidencia de género y número). Es la que establece el sustantivo con el artículo o los adjetivos que lo acompañan: la blanca paloma; esos libros viejos; el pronombre con su antecedente o su consecuente: a tus hijas las vi ayer; les di tu teléfono a los chicos; o el sujeto con el atributo, con el predicativo o con el participio del verbo de la pasiva perifrástica: mi hijo es un santo; ella se encontraba cansada; esas casas fueron construidas a principios de siglo.

2. Concordancia verbal (coincidencia de número y persona). Es la que se establece entre el verbo y su sujeto: esos cantan muy bien. 
Potowski y Escobar (2015: 101) explican que «el español clasifica todos los sustantivos, animados e inanimados, en dos géneros: masculinos y femeninos», a diferencia del inglés que no diferencia morfemas de género, aunque sí existen casos de diferenciación léxica de género, por ejemplo: he (masculino) vs she (femenino), him (masculino) vs her (femenino), king (masculino) vs queen (femenino) o bull (masculino) vs cow (femenino). Rodríguez (2005: 799) dice que este tipo de sustantivos se distinguen genéricamente «por procedimientos léxicos, no morfemáticos», como también se ve en muchos ejemplos españoles como oveja (femenino) vs carnero (masculino), hombre (masculino) vs mujer (femenino) o toro (masculino) vs vaca (femenino). En su libro explica que «la mayoría de los sustantivos [...] de las lenguas romances tienen género fijo» (Loc. Cit.), concluyendo entonces que las variaciones morfemáticas de género tienen únicamente valor gramatical. Esta diferenciación entre género léxico y semántico se ve más claramente en la explicación de Grandgent (1907: 144) al señalar que los tres géneros del latín no dependían, en el fondo, del sexo o de la carencia de sexo; eran distinciones gramaticales y si las palabras perdían sus terminaciones diferenciadoras resultaba la confusión de géneros.

Pero no solo los sustantivos tienen flexión genérica y numérica en español sino que además los determinantes y adjetivos que acompañan al sustantivo español deben estar en concordancia con él, es decir, deben estar en el mismo género y número. En el siguiente ejemplo vemos como dos palabras diferentes -por ser variantes geográficas- que refieren a la misma realidad, computadora y ordenador, tienen distinto género y tanto el determinante como el adjetivo están en concordancia con el sustantivo:

La computadora vieja no funciona. (Singular femenino) (Det. + Sust. + Adj. $\left.+\mathrm{Adv} .+\mathrm{V}^{\mathrm{o}}\right)$ El ordenador viejo no funciona. (Singular masculino) $\left(\right.$ Det. + Sust. + Adj. $\left.+\mathrm{Adv} .+\mathrm{V}^{\mathrm{o}}\right)$

\section{ESTADO DE LA CUESTIÓN}

Un elemento clave para comprender la problemática del aprendizaje de concordancia pasa por saber cuándo un hablante adquiere este concepto. Gathercole (2002: 3) señala que los nativos de español reconocen qué es concordancia a los 31 meses de edad y son capaces de concordar a los 3-4 años. Los niños bilingües de 6-7 años concuerdan igual o con menor precisión que sus compañeros nativos. Esta investigadora realizó un experimento y concluyó que los bilingües, entre los que se incluyen los $\mathrm{HH}$, tardan considerablemente más en concordar si no tienen suficiente input lingüístico en casa, aunque no es posible dar una respuesta a la edad de adquisición de género en estos hablantes. Montrul y Potowski (2007: 302) están de acuerdo en que la adquisición de concordancia se da entre los 3-4 años en hablantes monolingües pero es imprecisa la edad de adquisición en niños bilingües. Estas autoras investigaron los resultados de 60 niños bilingües ( $\mathrm{LH}$ y L2) y 29 monolingües. Los datos mostraron que los monolingües apenas tienen errores de concordancia, y los hablantes de herencia y aprendientes de L2 tienen bastantes errores, teniendo más precisión los $\mathrm{HH}$ pues suelen recibir más input que los aprendientes de L2. La edad de adquisición de concordancia es un tema comprometido en el que ningún autor se ha puesto de acuerdo porque depende directamente de cuánto tiempo ha estado en contacto con la lengua española un aprendiente de L2 o un HH. Como explica Zuheros (2016: 3-4), aunque en un primer momento lo parezca, la edad no es un factor importante 
a la hora de aprender una lengua. La adquisición de concordancia depende del tiempo de exposición a la lengua y no de la edad, puesto que cada aprendiente de español como L2 o bilingüe comienza su aprendizaje a edades variables. En contraposición, Potowski y Escobar (2015: 85) defienden la importancia del factor edad a la hora de aprender una lengua, especialmente al pasar el periodo crítico de 12 años de edad, cuando la adquisición de una lengua pasa a considerarse aprendizaje como L2.

Fleissing (2009: 54) investigó el uso de concordancia en niños nativos y de L2 para concluir que, en niveles básicos de español como L2, el estudiante construye la lengua de forma similar a como lo haría en su L1 y, si no existen errores, se debe a la experiencia y el estudio del hablante y las transferencias positivas que realiza de su interlengua, influenciada, en muchos casos, de otras lenguas en las que también existe el factor género. Para Fleissing (2009: 59-60) también es necesario que las estructuras que difieren de la L1 y la L2 hayan sido aprendidas con anterioridad para no cometer errores de concordancia. No se pueden, por el contrario, establecer causas cualitativas o cuantitativas a este fenómeno pues son múltiples los factores por los que se ve afectado, tales como origen del hablante, su L1, tiempo de contacto con la lengua, contextos de habla, etc. Montrul (2008: 537) comparó los resultados de concordancia de género en hablantes de español como L1, L2 y LH. Concluyó que los nativos no tienen problemas de concordancia, pero los estudiantes de L2 tienen dificultades al producir oralmente. Quizá esto se deba a que la oralidad es una competencia en la que el hablante tiene menos control en comparación con la competencia escrita, en la que se pueden hacer correcciones, aclaraciones o pausas para pensar detenidamente lo que está produciendo. Al contrario que los L2, los HH fallan sistemáticamente al escribir pues su español es básicamente oral, debido a que la lengua formal y vehicular suele ser inglés, y no poseen ni las herramientas ni las estrategias necesarias para una escritura formal adecuada. Gil (2016: 15) también comparte esta teoría al defender que los $\mathrm{HH}$ no producen el género como los nativos sino que deben aprenderlo tal como hacen los L2, y de ahí sus problemas de concordancia.

Por otra parte, algunos autores explican que la dificultad para concordar está íntimamente relacionada con la morfología del español. En cuanto a la importancia de los elementos morfológicos en la adquisición y producción de concordancia, Pérez (1990: 74), Lieberman (2010: 1579) y Foote (2015: 344) defienden que si un marcador morfológico claro está gramaticalmente presente, más temprano se adquirirá la capacidad de concordar y se cometerán menos errores, aunque esto no signifique la corrección completa del problema.

También, cuanto mayor es el nivel educativo del hablante, menos errores cometerá. Esto explica cómo en los niveles básicos de español como L2 se tiende a la regularización morfológica de casos irregulares. Pastor (2001: 33) añade que los problemas de concordancia en aprendientes de español como L2 disminuyen en los niveles altos y son apenas existentes en los superiores. Por lo general, en los casos agramaticales utilizados predomina el uso masculino, pues se debe al género no marcado, mostrando así más atención a las características morfológicas que al propio imaginario léxico del hablante. Pero esto no es un problema para los hablantes nativos porque los rasgos morfológicos no constituyen un elemento relevante para que puedan concordar porque prestan más atención a su propio imaginario que a las características morfológicas. Sin embargo, Foote (2015: 334), a diferencia de sus compañeros, añade que los hablantes nativos de español 
están acostumbrados a la concordancia en género entre artículo, sustantivo y adjetivo; esta característica define precisión en el dominio de la lengua y les diferencia de los L2 y los HH. Lieberman (2010: 1576) coincide también al afirmar que no todas las lenguas tienen género gramatical y un aprendiente de L2 puede tener problemas en su adquisición justamente por la falta de costumbre y la necesidad de aprenderlas. Añade que esta característica de género presenta rasgos morfológicos difusos y el propio concepto de género gramatical es un problema en sí pues para algunos hablantes no tiene lógica que un objeto inanimado sea masculino o femenino. Su investigación precisa que los estudiantes de nivel intermedio todavía no dominan la concordancia española aunque la producen más y con mayor facilidad que en etapas precedentes.

Contradiciendo lo anterior, Montrul (2008: 533) afirma que la elección de género por parte de un hablante se debe al enlace que existe entre el concepto mental de género y su lexicón, no por medio de elementos morfológicos. Mientras que este enlace es completamente funcional en la L1, diferentes fenómenos ocurren durante el aprendizaje de una L2 o una LH, teniendo estos dos grupos objetivos y necesidades diferentes. Siguiendo esta estela de aprendizaje, Potowski (2005: 52-54) explica que la enseñanza de los morfemas gramaticales que conforman el género y ayudan al proceso adquisitivo de concordancia debe realizarse implícitamente en los hablantes de herencia y dejar la gramática explícita para otro tipo de aprendientes. En esta misma línea, Boyd (1975: 130) investigó la similitud del aprendizaje de español como L1 y L2. Vio que ambos aprendizajes pasan por etapas, pero ni tienen que ser las mismas, ni darse en el mismo orden o en el mismo tiempo. Con todo esto, lo que queda claro es que la temporización no es igual en todos los casos de aprendizaje del español. En lo que sí coinciden es que el hablante de cualquier lengua a una edad de adquisición temprana busca poder comunicarse más que la corrección morfológica; sin embargo, superada esta etapa en la que prima la comunicación, la L1 tiende a evolucionar más rápidamente que la L2. Desde el punto de vista de Sánchez-Sadek (1975: 8), el aprendiente de español como L2 tardará más en concordar que los nativos o los bilingües justamente porque, aunque la etapa comunicativa inicial es la misma, la L1 y la L2 se desarrollan con tiempos diferentes.

Se puede concluir que todos los autores están de acuerdo en que aunque algunas etapas adquisitivas se compartan, deben tenerse en cuenta otros aspectos lingüísticos, semánticos o fonológicos que hacen que no se den en el mismo tiempo. La concordancia es una etapa primordial en el aprendizaje de una lengua, pero esta etapa no comparte el mismo periodo en la L1, en la L2 o la LH. Serramitja (2012: 824) observa que la influencia de la L1 para el aprendizaje de una L2 es crucial, aunque también hay que tener en cuenta el proceso natural del aprendizaje, dividido en niveles de competencia, de la propia L2. Relacionando esta última teoría con los hablantes de herencia, Escobar y Potowski (2015: 101) añaden que «son varios los estudios que han documentado errores de concordancia de género gramatical en niños bilingües y en adultos, generalmente de la segunda generación», pudiendo deducirse que las etapas de adquisición de concordancia en la lengua de herencia difieren dentro de cada generación, con lo que no existiría una línea temporal de adquisición en los hablantes de herencia sino que habría tres, una por cada generación (si solo tenemos en cuenta G1, G2 y G3 y no las etapas intermedias G0.5, G1.5, G2.5, G3:1 y G3:2). Montrul (2018: 147-148) concluye que los estudios más importantes sobre concordancia y lengua de herencia defienden que tanto los hablantes de herencia como 
los estudiantes de L2 tienen problemas de concordancia en niveles bajos e intermedios, siendo más comunes en hablantes de herencia cuando los nombres son femeninos o no tienen marcadores morfológicos claros. Una vez que los $\mathrm{HH}$ obtienen una competencia alta en español, su nivel de concordancia es similar al de un hablante nativo.

Complementando los estudios citados con anterioridad, White et al. (2004: 127) llevó a cabo una investigación que responde a todas las cuestiones importantes referentes al español como L2 y sus etapas de adquisición. En ella se analizaron los casos de concordancia de grupos nominales en español formados por determinante, sustantivo y adjetivo comparando tres tipos de hablantes. El primer grupo estaba constituido por nativos de español; el segundo eran estudiantes de español cuya L1 era francés; y el tercero eran estudiantes de español cuya L1 era inglés. Los nativos fueron tomados como grupo de control para contrastar los resultados entre los otros dos grupos. Se seleccionaron grupos cuya L1 es francés e inglés porque en francés hay morfemas de género y en inglés no, lo que serviría como marcador para investigar si la concordancia se adquiere más fácilmente si en la L1 también hay variación, ya sea léxica o morfológica. Los resultados de la investigación concluyeron que el nivel de estudios juega un papel importante, concordando entre un $71 \%$ y un $76 \%$ en niveles de principiante hasta la concordancia del más del $98 \%$ de los casos de nivel avanzado. Las diferencias entre los hablantes de francés e inglés no son muy grandes, siendo un 5\% el más acusado, concluyendo que la adquisición y producción de concordancia en español no se ve afectada si la L1 del hablante también es una lengua con marcadores morfológicos de género y número. Se puede inferir que la concordancia se adquiere cuando el alumno tiene nivel intermedio al superar el $90 \%$ de los casos, llegando a niveles cercanos a los nativos (98\%) en niveles avanzados.

Si nos centramos en algunas características de la adquisición de concordancia a un nivel más local debemos tener en cuenta la afirmación de Neira (1980: 51) cuando analizó la evolución de la lengua a lo largo del tiempo. En su estudio observó cómo las normas lingüísticas son modificadas por ciertos grupos o individuos sin relación y luego se expande al resto de la población. Siguiendo con esta definición, Dralo (2012: 1) defiende que la adquisición del español varía de una persona a otra y la motivación personal es un elemento clave para su desarrollo. Por este motivo, para el aprendizaje de una L2 o LH también hay que considerar a los propios aprendientes, sus motivaciones y sus contextos, incluso los diferentes dialectos del español puesto que las gramáticas cambian de un país a otro y el léxico sufre variaciones, o acomodaciones geográficas, que pueden resultar en un cambio de género. Tenemos por ejemplo el caso de la palabra Internet, de origen inglés, que en la variante castellana es masculina (Internet funciona lento) y en la variante mexicana es femenina (Internet funciona lenta). Todo esto nos lleva a ver el fenómeno de concordancia variable del español desde una perspectiva múltiple que puede ir desde el dominio de la lengua, la transferencia de la L1 a la L2 o la LH y la propia variación natural de la lengua.

\section{METODOLOGÍA}

Los datos recogidos en este trabajo se enmarcan en el proyecto Spanish in Texas dirigido por la Dra. Belpoliti en la Texas A\&M University-Commerce (TAMUC). Los estudiantes de la clase graduada Spanish in the USA construyeron un corpus lingüístico realizando entrevistas semidirigidas a hablantes de herencia de primera, segunda y tercera 
generación del área norte de Dallas. El corpus se completó con otras entrevistas realizadas por estudiantes de semestres anteriores. Además, para esta investigación en concreto se ha requerido el uso de 2 entrevistas de G1 y 2 de G3 del corpus realizado por la Universidad de Houston (UH) para tener el mismo número de entrevistas analizadas con respecto a generación y género de los hablantes.

Se ha seleccionado este método porque la entrevista es una técnica común en investigaciones cualitativas. Tiene grandes beneficios para el estudio de las ciencias sociales, siendo de vital importancia la naturalidad para recoger la muestra. Como explica Hernández (2014: 188), las entrevistas muestran experiencias cotidianas de los individuos en un contexto natural, sin interferencia de elementos artificiales, es decir, dentro de los límites de una conversación con un desconocido, los entrevistados se encuentran en un entorno seguro en el que sus respuestas serán naturales al no ser considerada una técnica puramente científica e intrusiva.

Para realizar las entrevistas de esta investigación se siguieron dos pasos. En primer lugar, el entrevistado cumplimentó anónimamente un cuestionario con información biográfica tal como lugar de nacimiento propio, lugar de nacimiento de los padres, edad, y otros datos sociales lingüísticamente relevantes para un hablante de herencia. En segundo lugar, para la entrevista semidirigida se utilizó una plantilla con preguntas guía ordenadas por temas y usos gramaticales de dificultad estructural ascendente, es decir, la entrevista comienza con preguntas de ámbito descriptivo (ciudad de residencia, restaurante favorito, etc.) para terminar con preguntas de respuesta irreal (experiencias sobrenaturales, lugares de residencia deseables, etc...) o argumentada (problemas de la comunidad hispana en EE.UU. y posibles soluciones), que requieren del uso de estructuras más complejas tales como subjuntivo o conectores textuales específicos. Las conversaciones tuvieron una duración de entre 30 y 50 minutos y fueron grabadas en audio digital para su posterior trascripción ortográfica (no fonética) incluyendo marcas prosódicas, elementos vocales no léxicos, errores de producción corregidos, etc. (Torruella 1999: 27-28). El corpus de la TAMUC consta de 63 entrevistas divididas principalmente en G1, G2 y G3, género, nivel educativo, origen y edad. Para esta investigación se han analizado un total de 24 entrevistas (20 del corpus de la TAMUC y 4 del corpus de la UH) divididas por igual entre generación y género, es decir, ocho entrevistas de G1 (cuatro hombres y cuatro mujeres), ocho de G2 (cuatro hombres y cuatro mujeres) y ocho de G3 (cuatro hombres y cuatro mujeres). Se utilizaron 24 entrevistas porque en un primer estudio piloto sobre concordancia general se usaron 12. Se decidió doblar el número hasta 24 para conseguir una muestra más amplia y comparar posteriormente los resultados con la primera investigación piloto. Hay que recordar que para conocer perfectamente las características de una sociedad habría que entrevistar a todos y cada uno de los habitantes, y eso es imposible. Por ese motivo se buscan muestras representativas que reflejen esas características sociales lo más fielmente posible.

A la hora de recolectar datos, se ha prestado antención al número de ítems y sus casos de concordancia, además del lugar en el que aparecen dentro de la oración: preverbal, posverbal o aislado. En total se han recogido 403 ítems de concordancia entre artículo, sustantivo y adjetivo en veinticuatro hablantes de herencia de las tres generaciones. El número entre paréntesis detrás de cada ejemplo indica el orden en el que apareció el grupo nominal cuando se examinaron las entrevistas y la posición que tiene dentro de la hoja de análisis. 
Los cinco casos analizados son:

1. Concordancia plena: la primera cosa (5), una recesión económica (17), todos los días (25), un poco enfermo (30), etc.

2. No hay concordancia con el determinante: este mucha gente (28), las luz roja (129), la año pasado (246), todo los tiempo (285), etc.

3. No hay concordancia con el adjetivo: la gente americano (85), la mismo escuela (253), una edad chiquito (261), todo la gente (378), etc.

4. No hay concordancia ni con el determinante ni con el adjetivo: todo eso lugares (388), un nuevo aventura (385), el mismo caricatura (384), el base chiquito (274), etc.

5. Otros problemas, como el orden inadecuado de los elementos que componen el grupo, omisión de un elemento o sustitución de alguna categoría gramatical: el carro todo (398), las diferencias creencias (331), todo [el] mundo (226), la misma biblia (152; el hablante no quería indicar que fuera igual, se refería a que era la biblia misma), etc.

Existen numerosas combinaciones dentro de los grupos nominales, pero este estudio se centra única y exclusivamente en los formados por determinante, nombre y adjetivo. Se excluyen aquellos casos en los que se repiten constituyentes como, por ejemplo, dos adjetivos o dos determinantes (los gatos blancos y negros), además de otras categorías de palabras como adverbios, conjunciones o intensificadores como «o», «muy» o «más» (los gatos blancos o negros; los gatos más blancos; los gatos muy blancos). Se ha decidido no tomar en cuenta estos casos como precaución a la posible interferencia entre los constituyentes. Sería de recibo realizar otro estudio paralelo sobre si realmente la inclusión de otras categorías gramaticales influye o no en la concordancia y, de ser así, cómo. Además, solo se recogen aquellas palabras que tienen género gramatical marcado o género léxico. Por ejemplo, se han descartado ítems como un carro grande porque, aunque gramaticalmente la palabra grande es masculina en este contexto, no hay ningún tipo de marcador que indique si el hablante quiere usarla como palabra con género masculino o femenino.

\section{ANÁLISIS DE DATOS}

Los resultados obtenidos se analizan teniendo en cuenta las variables generación, género, tipo de concordancia y posición del ítem. Para comenzar, la Figura 1 muestra el número de ítems encontrados (403) divididos en las tres generaciones. El mayor uso de estructuras nominales de tres constituyentes se da en la G1 (232 ítems, 58\% del total), bajando a la mitad en la G2 (113 ítems, 28\% del total) para finalmente encontrar pocos casos en la G3 (58 ítems, 14\% del total). La escalera decreciente que se crea muestra claramente como al aumentar la generación, el uso de estructuras compuestas disminuye drásticamente y las estructuras lingüísticas se simplifican ya que en dos generaciones se utilizan un $75 \%$ menos (de 232 usos a 58 usos). Esto se debería al desgaste que sufre el español de herencia a la par que aumenta la competencia en inglés. 


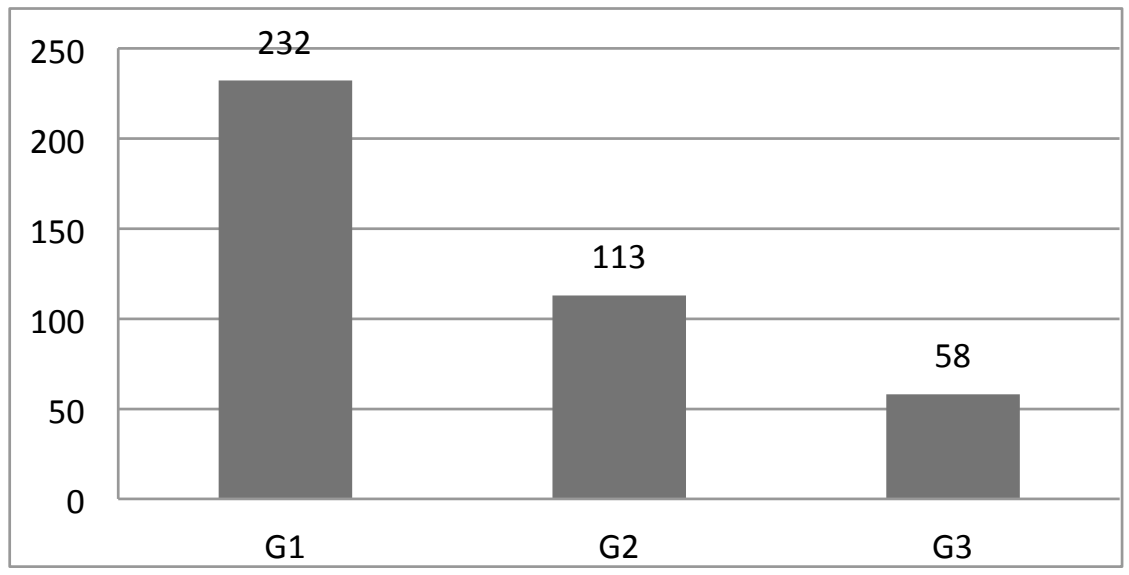

Figura 1. Ítems por generación

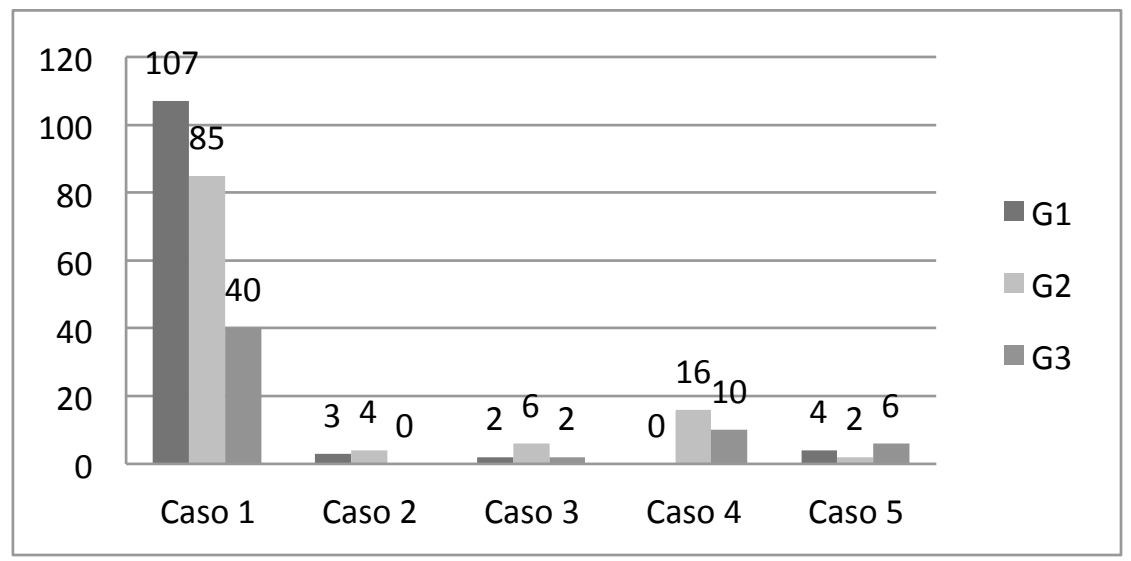

Figura 2. Ítems por caso y generación

La Figura 2 muestra el número de casos de concordancia relacionados con la generación. La aparición del Caso 1 (concordancia plena) es superior con respecto al resto: de un total de 116 casos en la G1, 107 tienen concordancia plena, por lo que la concordancia no es un problema en la G1. Si nos fijamos más detalladamente, la G2 contiene todos los casos estudiados, destacando sobre las demás generaciones en las formas agramaticales a excepción del Caso 5 (otros problemas), que la G3 está lígeramente por encima, seguido de la G1 y después de la G2. De esta forma, los casos de concordancia plena en lengua de herencia están muy por encima que el resto de casos. Con un perfil similar al visto en la Figura 1, las tres generaciones se encuentran escaladas en número decreciente en el Caso 1, donde hay 22 puntos de diferencia entre G1 (107) y G2 (85), existiendo una diferencia más acusada, algo más del doble, entre la G2 (85) y la G3 (40). El perfil descendiente con- 
firma el uso decreciente de la Figura 1 y la consecuente pérdida del español. En el resto de casos no hay una diferencia tan grande, pudiendo destacar el Caso 4, sin concordancia ni con el determinante ni con el adjetivo, en el que no hay representación de la G1 debido a la competencia lingüística avanzada de los hablantes. Si además comparamos el Caso 4 con el resto de casos agramaticales, se ve un pico de uso protagonizado indiscutiblemente por la $\mathrm{G} 2$ con 16 puntos y la G3 con 10 puntos.

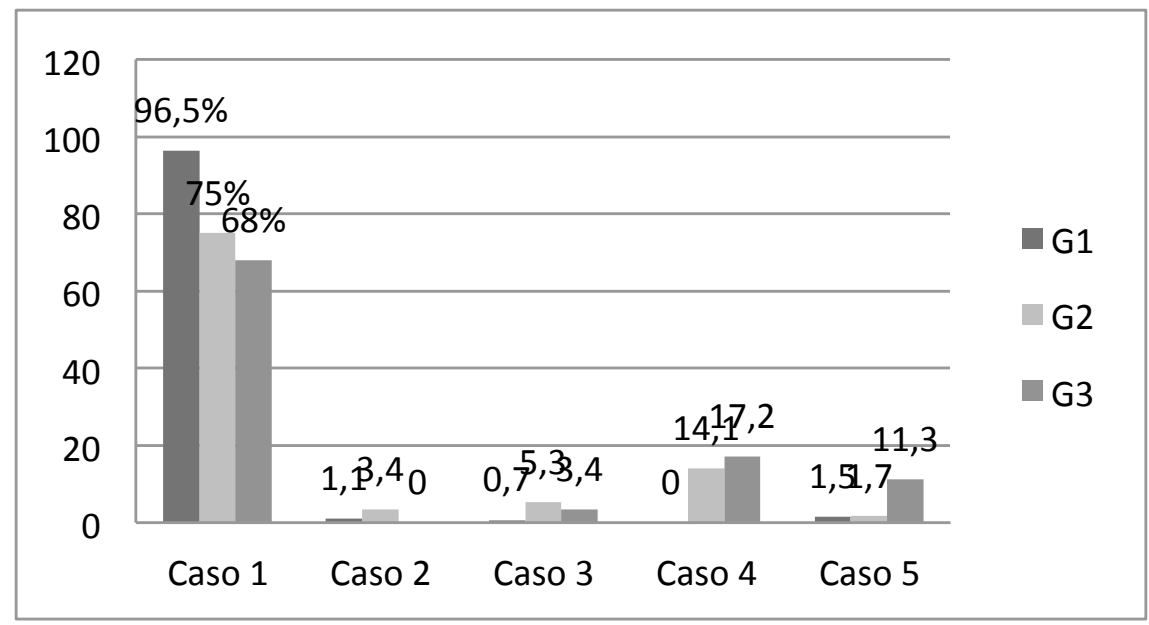

Figura 3. Ítems por caso y generación (porcentaje)

La Figura 3 muestra el porcentaje de casos encontrados en cada generación, es decir, los números de toda la G1 suman el 100\%, igual que los casos de la G2 y los de la G3. Con esta Figura se busca mostrar el porcentaje de uso de un tipo de caso dentro de esa misma generación. Otra vez se observa un perfil de escala decreciente por generaciones en el Caso 1, viendo como la concordancia plena en G1 roza el 100\% y en G2 tiene un 75\%, existiendo una diferencia cerca del $25 \%$ entre las dos primeras generaciones. Estas cifran muestran cómo la lengua se desgasta desde el primer cambio generacional. La concordancia baja hasta el $68 \%$ en G3, con una diferencia con la G2 poco acusada (7\%) y cerca de un $30 \%$ de diferencia con la G1. Al igual que en la Figura 2, en el Caso 4 hay un pico con respecto al resto de casos agramaticales, esta vez con un uso del $17 \%$ en la G3 y un $14 \%$ en la G2. Los casos de no concordancia constituyen un porcentage anecdótico en la G1 pero en la G2 y la G3 representan un porcentage bastante elevado que afecta a las morfosintaxis de los grupos nominales en la lengua oral.

Los datos de la Figura 4 se organizan teniendo en cuenta solamente los criterios de concordancia y no concordancia. Es visible el descenso del porcentaje de capacidad de concordancia (del 96.5\% en G1 a 75\% en G2 y 68\% en G3) frente al aumento de porcentaje en no concordancia (del 3.5\% en G1 al 24.7 en G2 y 32\% en G3). La escalera que se forma refleja el descenso de concordancia (mostrado anteriormente en el Caso 1 de la Figura 3) y el aumento de usos agramaticales según avanza la generación. Si comparamos estos porcentajes con los ítems totales de la Figura 1, llegamos a la conclusión de que la 
G3, además de utilizar menos la estructura nominal de tres elementos, comete porcentualmente más casos agramaticales que la G1 y la G2 juntas. Si observamos detenidamente, el salto porcentual de diferencia entre G1 y G2 es del 20\%, y entre G2 y G3 es de solo $7 \%$. Esto indica que la G1 está alejada de la G2 y G3, como si formaran un único grupo, y estas dos últimas muestran pocas diferencias en los casos de concordancia. Lo mismo podría aplicarse en los casos de no concordancia, en los que la G1 solo tiene un 3.5\%, muy alejado del $24.7 \%$ de la G2 y del $32 \%$ de la G3. Vemos otra vez como la diferencia entre G1 y G2 está cerca del 20\% mientras que entre la G2 y la G3 apenas es del $8 \%$. Visualmente se ven dos grupos, G1 vs G2 y G3, y no tres generaciones. Estos dos grupos representan el gran salto lingüístico que ocurre entre la G1 y la G2, cuando el español pierde fuerza frente al inglés, que será la nueva lengua dominante frente a la anterior competencia nativa en español.

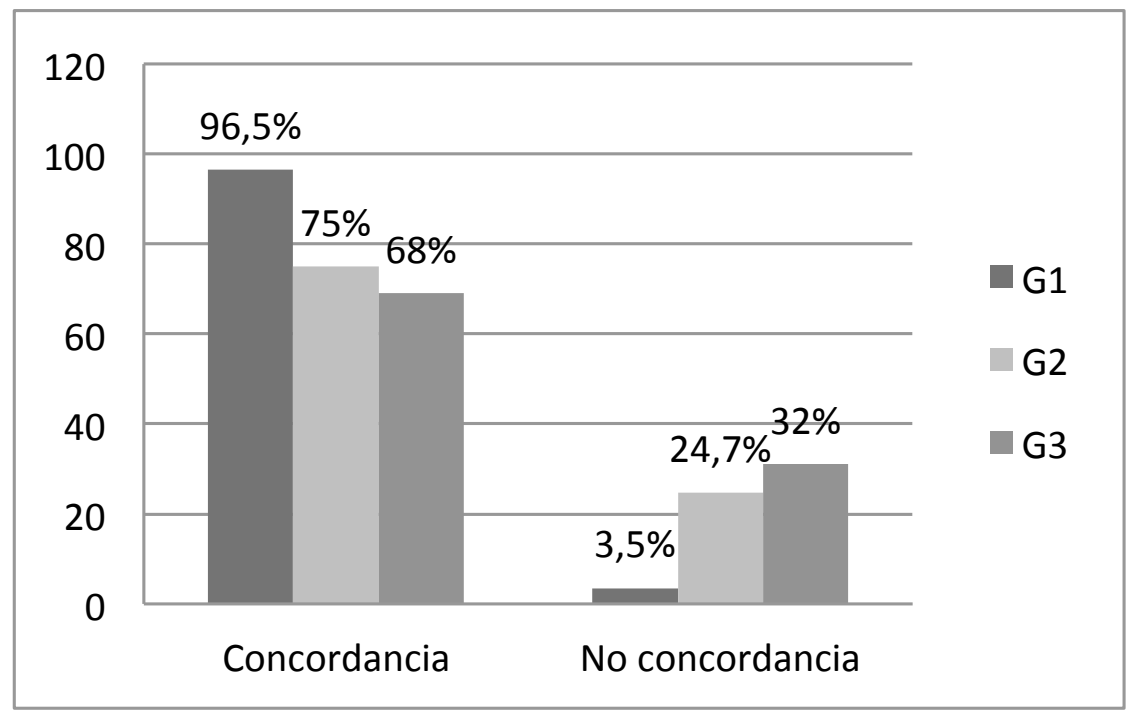

Figura 4. Concordancia vs. no concordancia (porcentaje)

En la Figura 5 se muestra la posición de los ítems con respecto al verbo. Del total de 403 ítems encontrados en las 24 entrevistas, 92 (22.7\%) están antes del verbo [una buena amiga para mí es alguien... (168)], 291 (71.8\%) están después del verbo [crecí en una ciudad pequeña (164)] y 20 (4.9\%) están aislados [por Cristo eres salvo, las experiencias religiosas, yo hace años (74)]. Existe una clara mayoría de ítems posverbales, seguido de casos preverbales para terminar con una mínima representación de casos aislados. Además, como se ha visto en figuras anteriores, todas las generaciones se encuentran perfectamente escaladas de G1 a G3 en cada uno de los tres lugares con respecto al verbo. El uso posverbal destaca sobre el resto, viendo como se prefiere esta posición. El uso preverbal tiene números destacables, con 55 casos en la G1, 24 en la G2 y 13 en la G3, dejando muy atrás a los aislados, con 12 en la G1, 6 en la G2 y solamente 2 en la G3. De esta forma se da, una vez más, una escala decreciente de la G1 a la G3. 


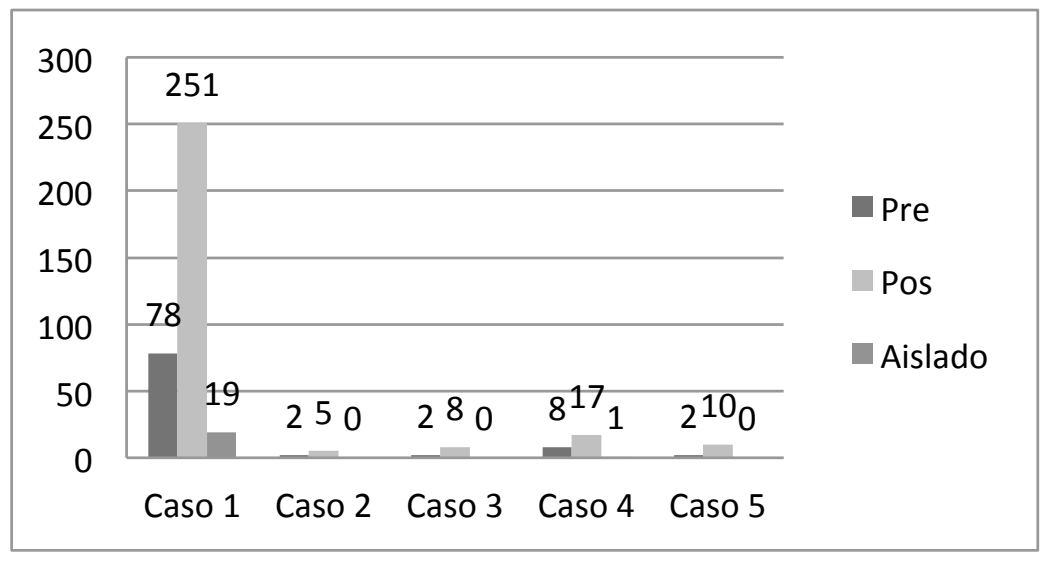

Figura 5. Posición del ítem

La Figura 6 muestra que en todos los casos prevalece la posición posverbal. La posición aislada es casi exclusiva del Caso 1, encontrando únicamente un único Caso 4, que está ausente en el resto de formas agramaticales. La suma total de casos agramaticales posverbales (40) es más del doble que la suma de agramaticales preverbales (14), con lo que es más común encontrar errores en posiciones posverbales. Sin embargo, la tendencia general en todo el estudio indica que el uso posverbal está generalizado, y si se encuentran más errores que en otras posiciones, es porque también se usa más. Una vez comenzado el enunciado hay más información en el predicado que en el sujeto y, por consiguiente, el número de estructuras después del verbo es superior.

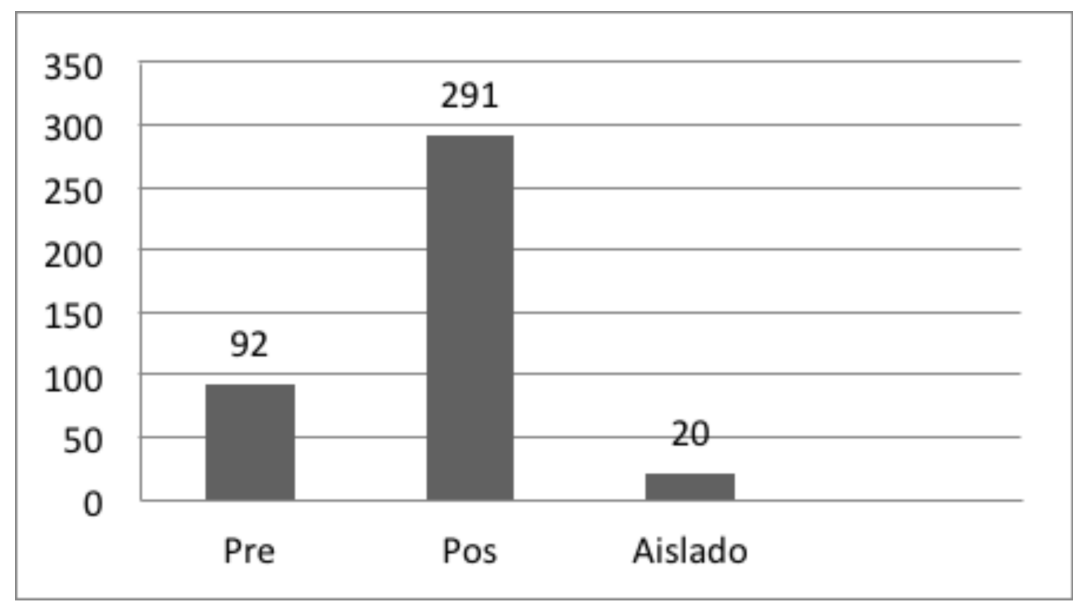

Figura 6. Posición de los casos 


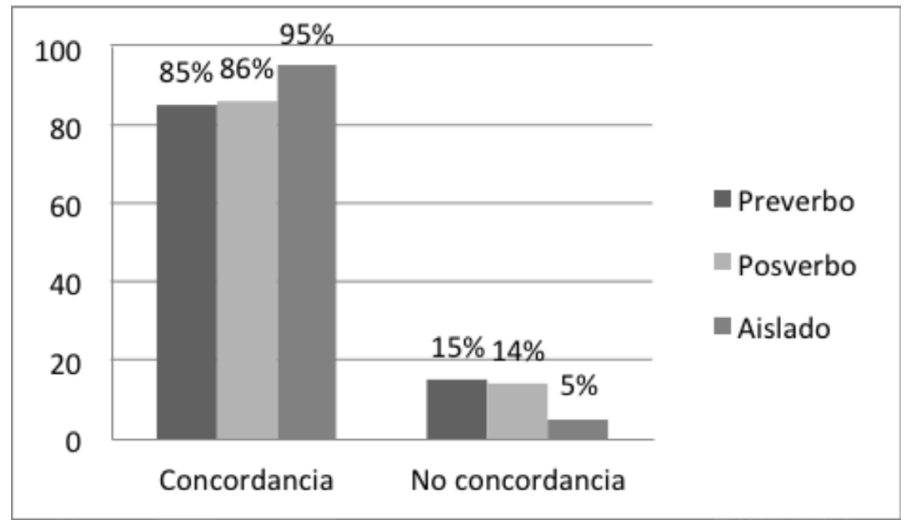

Figura 7. Concordancia por posición (porcentaje)

Si se tiene en cuenta el porcentaje de concordancia y no concordancia dependiendo de la posición del ítem con respecto al verbo, la Figura 7 muestra como, aunque la posición posverbal es superior con respecto al resto -Figura 5-, el porcentaje relativo de concordancia es similar en los casos preverbales y posverbales, con un $85 \%$ y un $86 \%$ respectivamente. Los casos aislados, aunque solo hay 20 en el estudio, tienen un porcentaje de concordancia muy elevado en su uso (95\%). Con esta Figura podemos afirmar que la posición del ítem con respecto al verbo no es importate para su concordancia pues el porcentaje es, prácticamente, el mismo. Sin embargo, la concordancia de estructuras aisladas es muy superior a las estructuras dentro de enunciados más complejos.

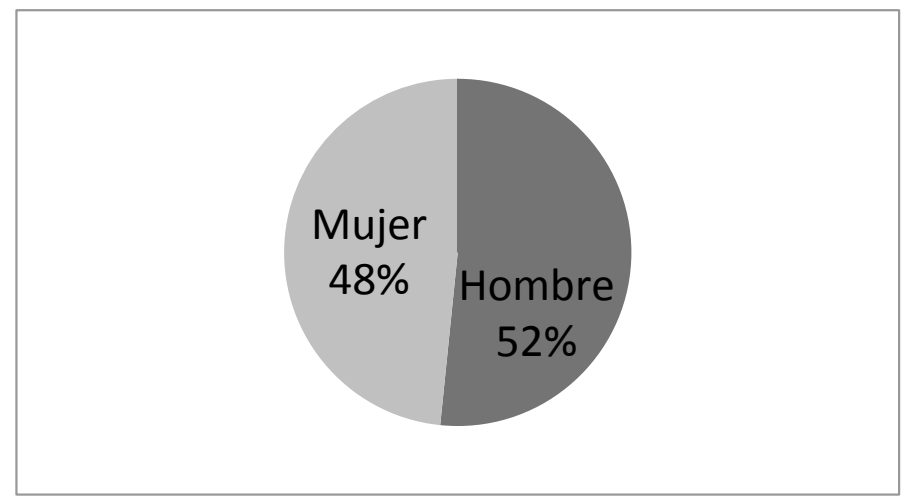

Figura 8. Ítems por género (porcentaje)

Con respecto al género, la Figura 8 muestra que apenas existen diferencias en la cantidad de ítems entre hombres y mujeres, aunque los hombres utilizan ligeramente más los grupos nominales de tres elementos, con una diferencia de solamente el 4\% (208 ítems en hombres y 195 en mujeres). Sin embargo, las mujeres prefieren esta estructura en la G1 con 131 
ítems, muy por encima del resto de generaciones, que juntas suman menos de la mitad, 64 ítems. Por otro lado, aunque el hombre también prefiere el uso en la G1, con 101 ítems, la diferencia con respecto a la G2 (76 ítems) no es tan acusada como en la mujer, aunque sí deja a la G3 (31 ítems) en una posición bastante inferior. Además, se encontró que tanto los hombres como las mujeres tienen el mismo número de ítems de cada caso salvo pequeñas excepciones. Hombres y mujeres concuerdan igual pues ambos tienen 174 casos de concordancia plena. También tienen los mismos números en el Caso 3, no concordancia con el adjetivo, y el Caso 5, otros problemas. Aunque están a la par en estos casos, el grupo masculino dobla a las mujeres en el Caso 4 (18 de hombres frente a 8 de mujeres), sin concordancia ni en el determinante ni en el adjetivo. A simple vista llegamos a dos conclusiones: los hombres utilizan la estructura lígeramente más que las mujeres, como se muestra en la Figura 8; y las mujeres concuerdan más que los hombres, independientemente del caso del que estemos hablando, aunque prefieren hacerlo en la G1.

Para terminar con la variable género, la posición de los ítems dependiendo del género varía poco. El uso posverbal tiene menos de un punto de diferencia, 35.8\% en hombres y $36.4 \%$ en mujeres, y el uso aialado medio punto, $2.7 \%$ en hombres y $2.2 \%$ en mujeres. Sin embargo, en la posición preverbal sí se encuentra una pequeña diferencia de tres puntos, $12.8 \%$ en hombres y $9.8 \%$ en mujeres, que no llega a ser una diferencia representativa.

\section{CONCLUSIONES}

Este trabajo que se ha llevado a cabo en la zona de Dallas, Texas, investiga ciertos usos específicos del español como lengua de herencia. Se analiza la concordancia en los grupos nominales constituidos por determinante, sustantivo y adjetivo utilizando material real recogido mediante entrevistas semidirigidas. Este estudio concluye que las generaciones juegan un papel importante tanto en el uso total de grupos nominales complejos como en el grado de concordancia de los mismos. Asimismo, la variable género de los hablantes y la posición con respecto al verbo no son importantes para definir error o acierto en la concordancia.

En el estudio se observa que el patrón de concordancia en estructuras de tres constituyentes no es un problema estandarizado dentro de la lengua de herencia. Los casos de concordancia plena constituyen el $93 \%$ de la muestra y los otros casos juntos suman el otro $7 \%$, con lo que la agramaticalidad no es un error extendido (Figura 1). Con todos los datos ya analizados, volvemos a las hipótesis lanzadas en la introducción.

1. Uso decreciente de grupos nominales de tres constituyentes según aumenta la generación: los datos confirman que según avanza la generación, el uso de grupos nominales desciende. La Figura 1 muestra que la G1 utilizó un total de 232 grupos, la G2 utilizó 113 y la G3 apenas 58, casi una cuarta parte menos que la G1. Esto se debe a la disminución de la competencia lingüística de los hablantes de herencia cuando, según Potowski y Escobar (2015: 25), el inglés desplaza al español comúnmente en la G3.

2. Descenso porcentual de concordancia según aumenta la generación: los datos confirman la hipótesis. Aunque los casos de concordancia completa sigue muy por encima que los del resto juntos, según avanza la generación, el porcentaje de casos que concuerdan se acerca al de los casos con errores. La Figura 2 muestra que en 
la G1 hay 107 casos de concordancia frente a 9 del resto de errores. En la G2 hay 85 casos de concordancia frente a 28 del resto de errores. En la G3 hay 40 casos de concordancia frente a 18 del resto de errores. Además, en la Figura 4 se muestra una escalera descendente de concordancia ( $96.5 \%$ en G1, 75\% en G2 y $68 \%$ en G3) y una ascendente de no concordancia (3.5\% en G1, 24,7\% en G2 y 32\% en G3) según avanza la generación del hablante.

3. Predominio del uso posverbal: esta hipótesis ha quedado confirmada por los datos obtenidos. En la Figura 5 se ve que la mayoría de ítems se encuentran en posición posverbal con mucha diferencia con respecto a la posición preverbal y aislada. La Figura 6 muestra que los ítems no solo aparecen en posición posverbal en todos y cada uno de los casos sino que el Caso 1, concordancia plena, es el más alto con un total de 251 ítems encontrados de un total de 403. Además, la Figura 7 muestra que el porcentaje de ítems con respecto a la posición del verbo es importante para una adecuada concordancia, encontrando una diferencia de 1 punto entre la posición preverbal (85\%) y posverbal (86\%), y existiendo un $95 \%$ de concordancia en los casos aislados.

4. Igualdad de resultados entre hombres y mujeres: se confirma esta hipótesis. En la Figura 8 se ve que tanto hombres (52\%), como mujeres, $(48 \%)$, hacen el mismo uso total del grupo nominal estudiado. Lo mismo ocurre cuando se observa que existen los mismos perfiles a lo largo de los casos estudiados sin importar el género. Con estos datos llegamos a la conclusión de que no hay diferencias entre los dos géneros.

Respondiendo a la pregunta inicial de cuándo sería el momento idóneo para la enseñanza de concordancia nominal en clases de español de herencia, viendo los datos obtenidos en esta investigación, la respuesta es relativamente sencilla. En primer lugar, los profesores deben enfatizar su enseñanza en la G2. La Figura 2 muestra que no hay realmente un problema de concordancia en la G1 y la gran pérdida de concordancia comienza en la G2. Es necesario intervenir en este momento para cortar el problema y no transferir la forma no gramatical a la siguiente generación. En segundo lugar, el porcentaje más alto de concordancia plena se encuentra en la producción aislada. La concordancia realizada de esta forma es un $11 \%$ más alta que en las demás. Los profesores deberían aprovecharse de este factor creando y practicando con grupos nominales aislados para ayudarles en su construcción, paso a paso, hasta que el estímulo deseado sea contextualizado y el porcentaje total de concordancia aumente.

Ha de entenderse que las necesidades de los hablantes de herencia no son las mismas que los aprendientes de L2. Ming (2010: 31) defiende que, aunque los métodos de enseñanza para $\mathrm{HH}$ evolucionan rápido, no se ha construido una base sólida sobre la que asentarse. Este autor insta al uso del acercamiento tipo top-down, desde el texto hasta segmentos más pequeños, como los morfemas de género y número. Afirma que, aunque haya problemas para concordar en género y número, no es un tema prioritario y el contenido textual y los elementos supralingüísticos son más importantes pues sí pueden interferir en la comunicación. En la misma línea educativa, Burgo (2015: 227) critica que se han implementado fórmulas de enseñanza de L2 en LH y los resultados no son satisfactorios debido a las diferentes necesidades de los dos grupos. Sin embargo, existen tres modelos de enseñanza funcionales: instrucción gramatical implícita, ciclos de input y output, y 
respuesta interactiva. No podemos esperar que los $\mathrm{HH}$ aprendan la concordancia igual que la L2, sino que hay que enseñarles las normas de forma más natural e intuitiva durante la G2, pues es donde más pérdida se ha encontrado. Con todo, Valdés y Parra (2018: 316-317) consideran que, aunque hay avances significativos en el campo de la enseñanza de lenguas de herencia, se deben estandarizar los criterios de los programas de herencia para trabajar bajo un mismo marco metodológico además de considerar los resultados para aprender de ellos y seguir mejorando la instrucción. Básicamente, se debe hacer más autocrítica y unificar bajo un mismo currículo pedagógico los métodos de enseñanza para alinear objetivos y resultados.

Aun llevando un proceso concienzudo, el trabajo tiene dos limitaciones que se solucionarán en futuras revisiones.

1. El problema más importante viene dado por el material utilizado, es decir, el corpus de entrevistas. Al haber sido realizado en su mayoría por estudiantes graduados con experiencia limitada en el campo de la investigación, existieron pequeños problemas a la hora de seguir un criterio único de codificación. Algunas entrevistas no estaban transcritas adecuadamente y otras no llegaban al tiempo mínimo requerido. Fue un problema esperable que se subsanó in situ escuchando de nuevo las entrevistas, repasando las transcripciones proporcionadas en busca de posibles errores metodológicos y utilizando las entrevistas cedidas por la Universidad de Houston. Todos los estudiantes que colaboraron en la creación del corpus estuvieron trabajando en su mejora académica para solucionar estos problemas.

2. Puede existir un posible problema de interpretación con el Caso 4. Realmente no se puede identificar si los hablantes tienen problemas al concordar el género del determinante y el adjetivo con el sustantivo o si realmente identifican el sustantivo con otro género y para ellos sí concuerda plenamente en su interlengua.

Para futuros estudios se tendrán en cuenta estos problemas además de la posibilidad de incluir y analizar las generaciones intermedias G0.5, G1.5 y las dos subdivisiones existentes en la G3 (G3:1 y G3:2). Potowski y Escobar (2015: 22) explican las características de estas generaciones de la siguiente manera:

1. La generación 0.5 (G0.5) llegó a EE.UU. con menos de 5 años. Tiene el español como lengua nativa y el inglés es la nueva lengua.

2. La generación 1.5 (G1.5) llegó a EE.UU. entre los 6 y los 8 años. El español es su lengua nativa. El inglés es la nueva lengua y la competencia dependerá del tiempo que permanezca en el país.

3. La generación 3:1 (G3:1) es una persona que nació en los EE.UU. y tiene un progenitor G1 y otro G2. El español suele ser la lengua débil y el inglés la lengua fuerte.

4. La generación 3:2 (G3:2) es una persona que nació en los EE.UU. y tiene dos progenitores de la G2. El español suele ser una lengua muy débil y el inglés la lengua dominante.

Para concluir, sería interesante ver una Figura en la que se muestre la evolución de concordancia en grupos nominales a través de todas y cada una de las generaciones. Ade- 
más, podrían subdividirse los casos agramaticales encontrados y diferenciar si se deben a motivos de género o número para concretar más aún los resultados y obtener resultados complementarios.

\section{REFERENCIAS BIBLIOGRÁFICAS}

Bosque, I. (1999). “El nombre común”. En Bosque, I. y V. Demonte (dirs.). Gramática descriptiva de la lengua española. Madrid: Espasa, pp. 3-76.

Boyd, P. A. (1975). "The development of grammar categories in Spanish by anglo children learning a second language", Tesol Quarterly, 9(2), pp. 125-135.

Burgo, C. (2015). "Grammar teaching approaches for heritage learners of Spanish", En Proceedings of Central States Conference on the Teaching of Foreign Language. Minneapolis: Johnson Litho Graphics of Eau Claire, pp. 217-234.

Cervantes, J. y A. Rodríguez. (2015). El perfil de la población de origen mexicano en Estados Unidos. México: Centro de Estudios Monetarios Latinoamericanos (CEMLA).

Domitrescu, D. (2014). "La alternancia de lenguas como actividad de imagen en el discurso hispanounidense", Sociocultural Pragmatics, 2(1), pp. 1-34.

Demonte, V. (1999). "El adjetivo: clases y usos. La posición del adjetivo en el sintagma nominal". En Bosque, I. y V. Demonte (dirs.). Gramática descriptiva de la lengua española. Madrid: Espasa, pp. 129-216.

Dralo, A. (2012). "The logical problem of second language acquisition”. En Proceedings of International Conference: The future of Education (2nd Ed.). Florence: Simonelli Editore.

Fairchild, S. y J. Van Hell (2015). "Determiner-noun code-switching in Spanish heritage speakers", Bilingualism: Language and Cognition, 20(1), pp. 1-12.

Faussart, C., Jakubowicz, C. y M. Costes (1998). "Gender and number processing in spoken French and Spanish", Rivista di Linguistica, 11(1), pp. 75-101.

Fleissing, D. (2009). El análisis de los errores de concordancia de los estudiantes universitarios angloparlantes: Un estudio del aprendizaje del español como segunda lengua (Unpublished master dissertation). Universidad de Michigan, MI.

Foote, R. (2015). "The production of gender agreement in native and L2 Spanish: The role of morphological form”, Second Language Research, 31(3), pp. 343-373.

Gathercole, V. M. (2002). "Grammatical gender in bilingual and monolingual children: A Spanish morphosyntactic”. En K. Oller y R. E. Eilers (dirs.), Language and literacy in bilingual children. Clevedon: Multilingual Matters, pp. 207-219.

Gil, A. (2016). "El género gramatical en español entre los hablantes de herencia y los hablantes nativos", Entrehojas: Revista de Estudios Hispánicos, 6(1), Artículo 6.

Grandgent, C. H. (1907). An introduction to vulgar latin. Boston: D.C. Heath \& Co.

Hernández, R. (2014). "La investigación cualitativa a través de entrevistas: Su análisis mediante la teoría fundamentada", Cuestiones Pedagógicas, 23, pp. 187-210.

Laca, B. (1999). "Presencia y ausencia de determinante". En Bosque, I. y V. Demonte (dirs.). Gramática descriptiva de la lengua española. Madrid: Espasa, pp. 891-928.

Lieberman, D. (2010). “Adquisición del género gramatical en español L2. ¿En qué estadio se fija el parámetro?”. En Proceedings of IV Congreso Internacional de Letras. Buenos Aires: UBA, pp. 1576-1580.

Lipski, J. M. (2005). “Code-switching or borrowing? No sé so no puedo decir, you know”. En Selected Proceedings of the Second Workshop of Spanish Sociolinguistics, pp. 1-15.

Martínez, J. A. (1999). "La concordancia". En Bosque, I. y V. Demonte (dirs.). Gramática descriptiva de la lengua española. Madrid: Espasa, pp. 2695-2786. 
Miller, L. y C. Gutiérrez (2019). "Los hablantes de herencia y la norma lingüística del español estadounidense", Glosas, 9(10), pp. 32-49.

Ming-Hsuan, W. y C. Tzu-Min (2010). "Heritage language teaching and learning through a macroapproach", Working Papers in Educational Linguistics, 25(2), pp. 23-33.

Montrul, S. (2015). The acquisition of heritage language. Cambridge University Press.

Montrul, S. (2018). "Morphology, syntax, and semantics in Spanish as a heritage language". En K. Potowski (ed.). The Routledge handbook of Spanish as a heritage language. Londres y Nueva York: Routledge, pp. 145-163.

Montrul, S., Foote, R. y S. Perpiñán (2008). "Gender agreement in adult second language learners and Spanish heritage speakers: The effects of age and context of acquisition", Language Learning, 58(3), pp. 503-553.

Montrul, S. y K. Potowski (2007). "Command of gender agreement in school-age Spanish-English bilingual children", The International Journal of Bilingualism, 11(3), pp. 301-328.

Moreno, O. (2009). "La enseñanza del español a los hispanos de EE.UU.: desafíos y respuestas prácticas en la universidad norteamericana", El Profesor de Español ELE, 2, pp. 681-697.

Neira, J. (1980). "Cambio lingüístico y cambio de lenguas", El Basilisco, 9, pp. 47-53.

Pastor, S. (2001). "La concordancia en la interlengua del español como lengua extranjera". En Pastor, S. y V. Salazar (eds.). Tendencias y Líneas de Investigación en Adquisición de Segundas Lenguas, Anexo 1 de E.L.U.A. Alicante: Universidad de Alicante.

Pérez, M. (1990). “Cómo determinan los niños la concordancia de género?: Refutación de la teoría del género natural”, Infancia y Aprendizaje, 50, pp. 73-91.

Potowski, K. (2005). Fundamentos de la enseñanza del español a hispanohablantes en los EE.UU. Madrid: Arco Libros.

Potowski, K. (2018). "Spanish as a heritage/minority language. A multifaceted look at ten nations". En $\mathrm{K}$. Potowski (ed.). The routledge handbook of Spanish as a heritage language. Londres y Nueva York: Routledge, pp. 1-14.

Potowski, K. y A. M. Escobar (2015). El español de los Estados Unidos. Cambridge University Press. Real Academia Española. 2014. Diccionario de la lengua española, 23 ed. http://www.rae.es/rae.html Rigau, G. (1999). "La estructura del sintagma nominal: los modificadores del nombre". En Bosque, I. y V. Demonte (dirs.). Gramática descriptiva de la lengua española. Madrid: Espasa, pp. 311-362.

Rodríguez, B. (2005). El género: del latín al español: los nuevos géneros del romance. Universidad de León, Secretariado de publicaciones.

Ronquest, R. y R. Rao (2018). "Heritage Spanish phonetics and phonology". En K. Potowski (ed.). The routledge handbook of Spanish as a heritage language. Londres y Nueva York: Routledge, pp. 164-177.

Sánchez-Sadek, C., Kiraithe, J. y H. Villarreal (1975). "The acquisition of the concept of grammatical gender in monolingual and bilingual speakers of Spanish", Colloquium on Hispanic linguistics, pp. 131-149.

Serramitja, L. (2012). La adquisición de los determinantes del español por parte de hablantes chinos, rusos y rumanos: Codificación de errores. En Proceedings of XXIII Congreso Internacional de la ASELE. Girona: ASELE, pp. 819-826.

Torruella, J. y J. Listerri (1999). "Diseño de corpus textuales y orales". En Blecua, J. M., Clavería, G., Sánchez, C. y J. Torruella (eds.). Filología e informática. Nuevas tecnologías en estudios filológicos. Barcelona: Universidad Autónoma de Barcelona - Editorial Milenio.

Valdés, G. (2001). "Heritage language students: Profiles and possibilities". En Kreeft, J., Ranard, D. y S. McGinnis (dirs.). Heritage languages in America. Preserving a national resource. USA: CAL., pp. 37-77. 
Valdés, G. y M. L. Parra (2018). “Towards the development of an analytical framework for examining goals and pedagogical approaches in teaching language to heritage speakers". En K. Potowski (ed.). The routledge handbook of Spanish as a heritage language. Londres y Nueva York: Routledge, pp. 301-330.

White, L., Valenzuela, E., Kozlowska-McGregor, M. y Y. Leung (2004). "Gender and number agreement in nonnative Spanish", Applied Psycholinguistics, 25, pp. 105-133.

Zuheros, L. y V. Marrero (2016). "Adquisición de la gramática del español en niños y adultos sinohablantes. La concordancia nominal”, SINOELE, 17, pp. 1030-1035. 
